Research Article

\title{
Sex Difference of Ribosome in Stroke-Induced Peripheral Immunosuppression by Integrated Bioinformatics Analysis
}

\author{
Jian-Qin Xie $\mathbb{D}^{1}{ }^{1,2,3}$ Ya-Peng Lu $\mathbb{D}^{1,3}$ Hong-Li Sun $\mathbb{D}^{1,3}$ Li-Na Gao $\mathbb{D}^{1,3}, 3$ Pei-Pei Song $\mathbb{D},{ }^{2,3}$ \\ Zhi-Jun Feng $\mathbb{D}^{3}{ }^{3}$ and Chong-Ge You $\mathbb{D}^{2,3}$
}

${ }^{1}$ Department of Anesthesiology, Lanzhou University Second Hospital, Lanzhou, Gansu 730030, China

${ }^{2}$ Laboratory Medicine Center, Lanzhou University Second Hospital, Lanzhou, Gansu 730030, China

${ }^{3}$ The Second Clinical Medical College of Lanzhou University, Lanzhou, Gansu 730030, China

Correspondence should be addressed to Chong-Ge You; youchg@lzu.edu.cn

Received 13 April 2020; Revised 8 October 2020; Accepted 18 November 2020; Published 7 December 2020

Academic Editor: Rudolf K. Braun

Copyright ( 2020 Jian-Qin Xie et al. This is an open access article distributed under the Creative Commons Attribution License, which permits unrestricted use, distribution, and reproduction in any medium, provided the original work is properly cited.

\begin{abstract}
Ischemic stroke (IS) greatly threatens human health resulting in high mortality and substantial loss of function. Recent studies have shown that the outcome of IS has sex specific, but its mechanism is still unclear. This study is aimed at identifying the sexually dimorphic to peripheral immune response in IS progression, predicting potential prognostic biomarkers that can lead to sexspecific outcome, and revealing potential treatment targets. Gene expression dataset GSE37587, including 68 peripheral whole blood samples which were collected within 24 hours from known onset of symptom and again at 24-48 hours after onset (20 women and $14 \mathrm{men}$ ), was downloaded from the Gene Expression Omnibus (GEO) datasets. First, using Bioconductor R package, two kinds of differentially expressed genes (DEGs) (nonsex-specific- and sex-specific-DEGs) were screened by followup (24-48 hours) vs. baseline (24 hours). 30 nonsex-specific DEGs (1 upregulated and 29 downregulated), 79 female-specific DEGs (25 upregulated and 54 downregulated), and none of male-specific DEGs were obtained finally. Second, bioinformatics analysis of female-specific DEGs was performed. Gene Ontology (GO) functional annotation analysis shows that DEGs were mainly enriched in translational initiation, cytosolic ribosome, and structural constituent of ribosome. Kyoto Encyclopedia of Genes and Genomes (KEGG) pathway enrichment analysis shows that the top 6 enrichment pathways are ribosome, nuclear factor--kappa B (NF-kappa B) signaling pathway, apoptosis, mineral absorption, nonalcoholic fatty liver disease, and pertussis. Three functional modules were clustered in the protein-protein interaction (PPI) network of DEGs. The top 10 key genes of the PPI network constructed were selected, including RPS14, RPS15A, RPS24, FAU, RPL27, RPL31, RPL34, RPL35A, RSL24D1, and EEF1B2. Sex difference of ribosome in stroke-induced peripheral immunosuppression may be the potential mechanism of sex disparities in outcome after IS, and women are more likely to have stroke-induced immunosuppression. RPS14, RPS15A, RPS24, FAU, RPL27, RPL31, RPL34, RPL35A, RSL24D1, and EEF1B2 may be novel prognostic biomarkers and potential therapeutic targets for IS.
\end{abstract}

\section{Introduction}

Stroke was the second largest cause of death worldwide after ischemic heart disease and the second most common cause of worldwide disability-adjusted life years (DALYs). There were 80.1 million prevalent cases of stroke, which $84.4 \%$ contributed ischemic stroke (IS) and 13.7 million new stroke cases globally in 2016 [1]. The mean global lifetime risk of stroke increased from $22.8 \%$ in 1990 to $24.9 \%$ in 2016 [2]. On average, stroke occurs every 40 seconds, and stroke caused death every 3 minutes and 42 seconds in the United States [3]. Stroke is characterized by significant sex differences. The difference of sex on various aspects of stroke, such as risk factors, epidemiology, incidence, pathogenesis, mortality, prognosis, clinical presentation, and response to treatment, has been extensively investigated in the past years. Although a large number of experiments in vivo have proved that estrogen has a protective effect on the brain $[4,5]$, there is suffcient clinic evidence that women more often had poor functional outcome compared with men, and this difference 
was not dependent on age $[6,7]$. For example, a study found that young female rodents have smaller infarct volume and better cerebral blood flow (CBF) than age-matched males, and these sex differences reverse with aging [8]. Meanwhile, in a large European multicenter prospective observational clinical cohort study, researchers found that female sex remained associated with poor functional 3-month outcome after adjustments for baseline differences through analyzing 9495 acute IS patients who were treated with intravenous thrombolysis (IVT), and this finding was not dependent on age and could not be explained by a higher bleeding risk or mortality rate in women [7]. This study is basically consistent with the results of a previous study. The previous study based 47209 patients (>18 years) with ischemic stroke or clinically defined transient ischemic attack found that women had a worse functional outcome at 3-month follow-up, but a lower mortality after correcting for confounders [6]. So, not only sex hormones but also sex chromosomes may contribute to the different outcome following stroke. Therefore, it is necessary to further study the sex specificity of stroke, so as to provide basis for accurate evaluation of prognosis and formulation of treatment strategies.

In recent years, more and more attention has been paid to the pathogenesis of stroke-induced immunodeficiency syndrome (SIDS) [9] and stroke-associated infection (SAI). The inflammatory response after stroke is an important way to remove the necrotic tissue of brain injury, but excessive inflammatory response can also cause secondary inflammatory damage. Although stroke-induced immunosuppression is a necessary protective feedback mechanism for the body to resist inflammatory injury, it is also an important risk factor for stroke patients, which could reduce their immune defense, increase the susceptibility to infection, lead to serious infectious complications, and increase stroke mortality. Therefore, the balance between proinflammation and antiinflammation is the crucial to the recovery of IS patients. However, little is known about whether stroke-induced immunodepression has difference between female and male.

Because of the dynamic nature of transcriptional regulation, RNA levels represent not only features encoded in the genome but also the influence of the environment [10]. Thus, some investigators used gene expression profiling as the starting point for biomarker discovery and identification of disease mechanisms [11]. Microarrays based on highthroughput platforms for the profiling of genome-wide expression emerge as a promising and efficient tool for inferring biological relevancy. It is especially suitable for the study of the dynamic development process of complex diseases such as IS [12]. However, few studies have been done on the sexually dimorphic to peripheral immune response in IS progression at RNA level. This study tried to screen out the sex-specific differentially expressed genes (DEGs) in the peripheral immune response in IS progression. Normally in sexually dimorphic study, sex-specific DEGs (male specific and female specific) are main research target. But to further explore the potential connection between sex-specific DEGs and nonsex-specific DEGs, two kinds of DEGs, nonsexspecific DEGs and sex-specific DEGs (male specific and female specific), were screened in this study.
In this study, we have downloaded one original microarray dataset GSE37587 from the Gene Expression Omnibus (GEO) datasets, including 68 peripheral whole blood samples which were collected within 24 hours from known onset of symptom and again at 24-48 hours after onset (20 women and 14 men). Using Bioconductor $\mathrm{R}$ package, nonsexspecific and sex-specific DEGs were got by follow-up (24-48 hours) vs. baseline ( 24 hours) with 30 nonsex-specific DEGs, 79 female-specific DEGs, and 0 male-specific DEGs. Then, Gene Ontology (GO) functional annotation and Kyoto Encyclopedia of Genes and Genomes (KEGG) pathway enrichment on female-specific DEGs were performed by the $\mathrm{R}$ software. Finally, the STRING online database protein-protein interaction (PPI) network was used to analyze the association of female-specific DEGs and discover the molecular interactions involved in IS progression. Meanwhile, according to the PPI network result, molecular complex detection (MCODE) modules was established and key gene analysis was performed by Cytoscape for identifying key genes of optimal significance. In conclusion, sex-specific DEGs associated with the IS progression were screened and an integrated analysis was conducted. Our study is aimed at identifying the gender dimorphic to peripheral immune response in IS progression, predicting potential prognostic biomarkers that can lead to sex-specific prognosis, and revealing potential treatment targets.

\section{Materials and Methods}

2.1. Microarray Data. The GSE37587 gene expression profile series matrix file(s) and SOFT formatted family file(s) were downloaded from GEO datasets (https://www.ncbi.nlm.nih .gov/geo/), which platform is the GPL6883 Illumina HumanRef-8 v3.0 expression beadchip. Robust Multichip Average (RMA) normalization collation had been done with the series matrix file(s), including background correction, quantile normalization, and summarization. A total of 68 peripheral whole blood samples were included in the dataset, which were collected from $n=34$, and magnetic resonance imaging (MRI) diagnosed IS patients $\geq 18$ years of age within 24 hours from known onset of symptom and again at 24-48 hours after onset. IS patients, whose ethnicity are Caucasian, included 20 women with mean age of $72.15 \pm 15.951$ years and 14 men with mean age of $71.64 \pm 13.054$ years.

2.2. Screening for DEGs. The analysis was carried out with the $\mathrm{R}$ language software (version 3.6.2). First, the dataset was normalized by $\log _{2}$ transformation. Second, the 24,526 probe IDs were converted into gene symbols according to the SOFT formatted family file(s). If multiple probes correspond to the same gene, only the probe with the highest mean expression value is retained, and 18631 genes were finally obtained. Third, the DEG analysis was performed using the Linear Models for Microarray Data (limma) package (http://www .bioconductor.org/). DEGs were selected with thresholds of $\mid \log _{2}$ (fold change) (FC) $\mid>0.6$ and adjusted $p$ value $<0.05$. Two kinds of DEGs were screened: (1) nonsex-specific DEGs by follow-up (24-48 hours) vs. baseline (24 hours) and (2) sex-specific DEGs by female follow-up (24-48 hours) vs. 
Table 1: Nonsex- and sex-specific DEGs.

\begin{tabular}{|c|c|c|}
\hline DEGs & Regulated & Gene symbol \\
\hline \multirow[b]{2}{*}{$\begin{array}{l}\text { Nonsex- } \\
\text { specific }\end{array}$} & Upregulated & CDC14B \\
\hline & Downregulated & $\begin{array}{c}\text { LY96, SDPR, IL8, COMMD6, C15orf15, RPL31, CCDC72, COX7B, EVI2A, RPL17, AKAP7, RGS18, EEF1B2, } \\
\text { RPL27, TXNDC, RPS24, RPS15A, HES4, CAPZA2, RPL34, BAZ2B, BIRC2, C14orf156, HINT1, ARL5A, } \\
\text { MBNL2, MASK, RPL39, RPL26 }\end{array}$ \\
\hline \multirow[b]{2}{*}{$\begin{array}{l}\text { Female- } \\
\text { specific }\end{array}$} & Upregulated & $\begin{array}{c}\text { CDC14B, FCGR3A, HLA-G, AMY1A, GIMAP7, PTPLAD2, USP49, HNRPA2B1, ZNF557, RABL2A, LDHB, } \\
\text { ARHGDIA, CTSW, PTPRCAP, FAM40B, CD3E, PPIB, LOC441743, RPL23AP13, IL2RB, MBD4, HSPB1, } \\
\text { SF3B4, LOC400986, DENR }\end{array}$ \\
\hline & Downregulated & $\begin{array}{c}\text { LY96, SDPR, IL8, COMMD6, C15orf15, CCDC72, RGS18, RPL31, COX7B, CAPZA2, C21orf7, RPL17, } \\
\text { TXNDC, EVI2A, RPL27, BAZ2B, RPS15A, EEF1B2, RPL34, RPS24, PFDN5, C14orf156, BIRC2, TMEM55A, } \\
\text { HIST1H4H, GPR160, FAU, HSH2D, MMD, ARL5A, OXR1, RPL35A, PCNP, KIAA0143, LSM1, DHRS9, } \\
\text { SLC44A1, PPM1B, SNRK, ANKRD49, CKS2, RPS14, RCOR3, TMEM126B, CYBRD1, CLEC4D, CCPG1, } \\
\text { MGC4677, WDFY1, AP1S2, DDIT3, ST8SIA4, SLC40A1, SNX16 }\end{array}$ \\
\hline \multirow{2}{*}{$\begin{array}{l}\text { Male- } \\
\text { specific }\end{array}$} & Upregulated & None \\
\hline & Downregulated & None \\
\hline
\end{tabular}

Abbreviation: DEGs: differentially expressed genes. Note: C15orf15 is also known as RSL24D1.

female baseline (24 hours) and male follow-up (24-48 hours) vs. male baseline (24 hours).

\subsection{Bioinformatics Analysis}

2.3.1. GO Enrichment Analysis of Female-Specific DEGs. For the sets of female-specific DEGs, GO analysis was performed using clusterProfiler in R package with pAdjustMethod = "BH,", $p$ valueCutoff $=0.05$, and qvalueCutoff $=0.2$ [13]. Then, we used the function simplify to remove the redundancy of enriched GO terms.

2.3.2. KEGG Pathway Enrichment Analysis of Female-Specific DEGs. KEGG pathway enrichment analysis was performed using clusterProfiler with pAdjustMethod = "BH,", and $p$ value $<0.05$ was considered statistically significant.

2.3.3. Integration of the PPI Network Analysis. To evaluate the interactive relationships among the female-specific DEGs, we mapped them to the STRING database (http://string-db.org/) (version 11.0), and only the interaction score $>0.4$ (medium confidence) was considered significant. Interactions in STRING are derived from five main sources: genomic context predictions, high-throughput lab experiments, (conserved) coexpression, automated text mining, and previous knowledge in databases. The PPI network was visualized by the Cytoscape software (version 3.7.2).

2.3.4. Functional Module Identification in the PPI Network. MCODE, molecular complex detection, a Cytoscape plugin, clusters a given network based on topology to find densely connected. MCODE was used to identify functional module in the PPI network constructed with the default settings of degree cutoff at 2 , node density cutoff at 0.1 , node score cutoff at $0.2, \mathrm{k}$-core at 2 , and maximum depth at 100 .

2.3.5. Selection of Key Genes. CytoHubba, a Cytoscape plugin, was used to get the top 10 key genes in the PPI network constructed with the highest MCC score.

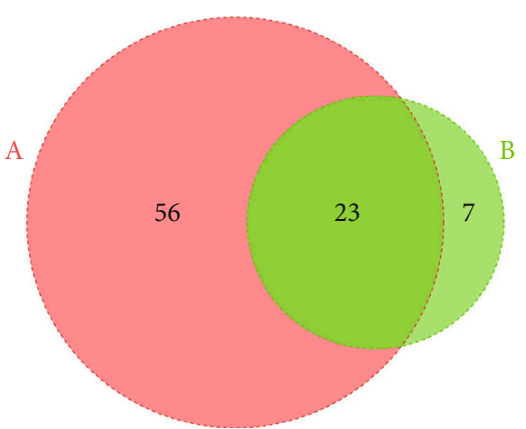

Figure 1: The coexpression distribution of female-specific and nonsex-specific DEGs. Red A represents female-specific DEGs. Green B represents nonsex-specific DEGs. DEGs: differentially expressed genes.

\section{Results and Discussion}

\subsection{Results}

3.1.1. Identification of Two Kinds of DEGs. The GSE37587 dataset contained 68 peripheral whole blood samples, which were collected from 20 women and 14 men within 24 hours (baseline) from known onset of IS symptom and again at 24-48 hours (follow-up) after onset. 30 nonsex-specific DEGs (1 upregulated and 29 downregulated) were obtained by comparison of 34 follow-up and 34 baseline samples. 79 female-specific DEGs (25 upregulated and 54 downregulated) were obtained by comparison of 20 female follow-up and 20 female baseline samples. And none of male-specific DEGs were obtained by comparison of 14 male follow-up and 14 male baseline samples. The details of all DEGs are shown in Table 1. The unique one upregulated gene in nonsex-specific DEGs is also upregulated in female-specific DEGs. The coexpression distribution of female-specific and nonsex-specific DEGs is shown in Venn diagram (Figure 1). Seven genes unique to nonsex-specific DEGs include AKAP7, RPL39, RPL26, MBNL2, HES4, MASK, and HINT1. The female-specific DEGs are shown in the volcano 


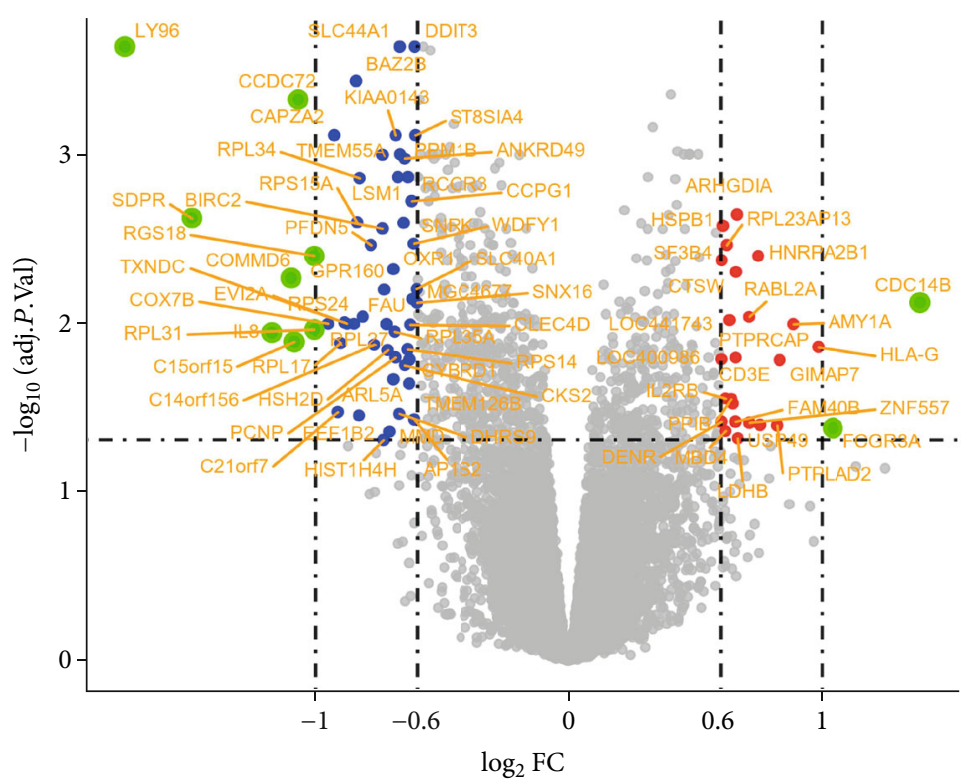

FIGURE 2: Female-specific differential expression of data between follow-up and baseline. The red points represent upregulated DEGs with $\log _{2} \mathrm{FC}>0.6$ and adjusted $p$ value $<0.05$. The blue points represent downregulated DEGs with $\log _{2} \mathrm{FC}<-0.6$ and adjusted $p$ value $<0.05$. The green points represent DEGs with $\left|\log _{2} \mathrm{FC}\right|>1$ and adjusted $p$ value $<0.05$. The grey points represent genes with $\left|\log _{2} \mathrm{FC}\right|<0.6$. The gene symbols of the DEGs are marked in orange. C15orf15 is also known as RSL24D1. DEGs: differentially expressed genes; FC: fold change.

plot (Figure 2), and the cluster heatmap is shown in Figure 3, which is, respectively, drawn by ggplot 2 package and heatmap function in the $\mathrm{R}$ software.

\subsubsection{Bioinformatics Analysis of Female-Specific DEGs}

3.1.3. GO Enrichment Analysis of Female-Specific DEGs. GO function annotation analysis was performed using clusterProfiler in R package, which was divided into three parts: biological process (BP), cell component (CC), and molecular function $(\mathrm{MF})$. Those results were considered statistically significant if $p$ value $<0.05$ and $q$ value $<0.2$. The redundancy of enriched GO terms was removed using the function simplify. The results are shown in Table 2 and Figures 4 and 5.

A total of 11 genes were enriched: two upregulated genes HSPB1 and DENR, nine downregulated genes RPS14, RPL35A, RPS24, RPL34, RPS15A, RPL27, RPL17, RPL31, and RSL24D1. The main BP enriched by the 11 genes are as follows: translational initiation, SRP-dependent cotranslational protein targeting to membrane, cotranslational protein targeting to membrane, nuclear-transcribed mRNA catabolic process, nonsense-mediated decay, and establishment of protein localization to endoplasmic reticulum. This indicates that in addition to the degradation of abnormal transcripts, these genes are mainly involved in protein biosynthesis: protein translation, postsynthesis secretion, and targeted transport in the cytoplasm. The most important CC enriched by all the nine downregulated genes is cytosolic ribosome. Meanwhile, the nine downregulated genes are enriched the only one MF result: structural constituent of ribosome; in other words, they encode the ribosomal structural protein.

3.1.4. KEGG Pathway Enrichment Analysis of Female-Specific DEGs. KEGG pathway enrichment analysis was performed using clusterProfiler in $\mathrm{R}$ package. A total of 18 genes are enriched in key signaling pathways, and all are downregulated genes except the only one CTSW that is upregulated. Six signaling pathways with higher rich factor than others are shown in Table 3 and Figure 6, which are ribosome, nuclear factor--kappa B (NF-kappa B) signaling pathway, apoptosis, mineral absorption, nonalcoholic fatty liver disease, and pertussis. However, there was no significant difference in pertussis. The network diagram was drawn using the Cytoscape software and shown in Figure 7.

\subsubsection{Integration of the PPI Network and Functional Module} Analysis. The STRING online database was used to analyze the 79 female-specific DEGs, and the PPI network was visualized using the Cytoscape software, as shown in Figure 8(a). Using MCODE in Cytoscape to analyze the PPI network, three functional modules are clustered, as shown in Figure 8(b).

The cluster 1 included 13 genes, FAU, RPL31, TMA7, RPS24, PFDN5, RPS14, RPL34, RPL35A, EEF1B2, RPL27, RSL24D1, RPL17, and RPS15A, related to ribosome function, involving translation, elongation, and folding in the process of protein synthesis. The cluster 2 includes three genes, $C D 3 E, I L 2 R B$, and DDIT3, related to T cell adaptive immune and apoptosis. The cluster 3 includes three genes, LY96, CXCL8 (also known as IL8), and BIRC2, related to NF kappa B signaling pathway.

3.1.6. Selection of Key Genes. The top key genes of the PPI network constructed were selected using CytoHubba in the Cytoscape software. The top key genes, which have higher MCC degree, including RPS15A, RPS14, RPL27, RPL35A, FAU, RPS24, RPL31, RPL34, EEF1B2 and RSL24D1, are 


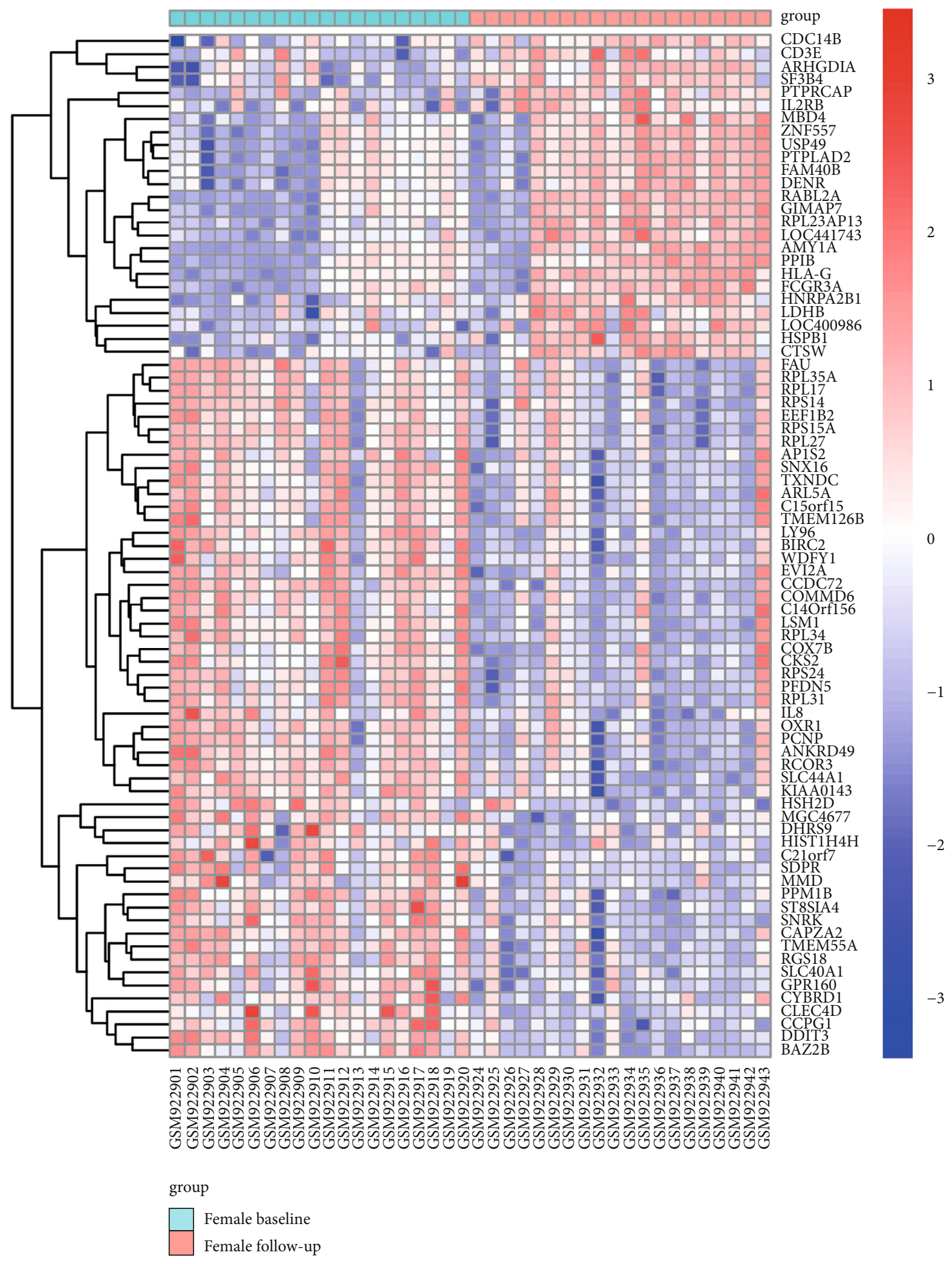

FIGURE 3: Cluster heatmap of the female-specific DEGs. Red indicates upregulation of gene expression. Blue indicates downregulation of gene expression. White indicates no significant change in gene expression. C15orf15 is also known as RSL24D1. 
TABLe 2: Enriched GO terms of female-specific DEGs.

\begin{tabular}{|c|c|c|c|c|c|c|c|}
\hline Category & ID & Term & $\begin{array}{l}\text { Gene } \\
\text { ratio }\end{array}$ & $\begin{array}{c}p \\
\text { value }\end{array}$ & $\begin{array}{c}p \\
\text { adjust }\end{array}$ & $\begin{array}{c}q \\
\text { value }\end{array}$ & Gene \\
\hline $\mathrm{BP}$ & 0006413 & Translational initiation & $10 / 73$ & $\begin{array}{c}3.90 E \\
-09\end{array}$ & $\begin{array}{c}3.80 E \\
-06\end{array}$ & $\begin{array}{c}3.63 E \\
-06\end{array}$ & $\begin{array}{l}\text { RPL34, RPS15A, HSPB1, RPS24, RPL27, } \\
\text { RPL31, RPL35A, RPL17, RPS14, DENR }\end{array}$ \\
\hline $\mathrm{BP}$ & 0006614 & $\begin{array}{l}\text { SRP-dependent cotranslational protein } \\
\text { targeting to membrane }\end{array}$ & $8 / 73$ & $\begin{array}{l}7.59 E \\
-09\end{array}$ & $\begin{array}{l}3.80 E \\
-06\end{array}$ & $\begin{array}{l}3.63 E \\
-06\end{array}$ & $\begin{array}{c}\text { RPL34, RPS15A, RPS24, RPL27, RPL31, } \\
\text { RPL35A, RPL17, RPS14 }\end{array}$ \\
\hline BP & 0006613 & $\begin{array}{l}\text { Cotranslational protein targeting to } \\
\text { membrane }\end{array}$ & $8 / 73$ & $\begin{array}{c}1.02 E \\
-08\end{array}$ & $\begin{array}{c}3.80 E \\
-06\end{array}$ & $\begin{array}{c}3.63 E \\
-06\end{array}$ & $\begin{array}{c}\text { RPL34, RPS15A, RPS24, RPL27, RPL31, } \\
\text { RPL35A, RPL17, RPS14 }\end{array}$ \\
\hline $\mathrm{BP}$ & 0000184 & $\begin{array}{l}\text { Nuclear-transcribed mRNA catabolic } \\
\text { process, nonsense-mediated decay }\end{array}$ & $8 / 73$ & $\begin{array}{c}2.18 E \\
-08\end{array}$ & $\begin{array}{l}4.62 E \\
-06\end{array}$ & $\begin{array}{l}4.41 E \\
-06\end{array}$ & $\begin{array}{c}\text { RPL34, RPS15A, RPS24, RPL27, RPL31, } \\
\text { RPL35A, RPL17, RPS14 }\end{array}$ \\
\hline BP & 0072599 & $\begin{array}{l}\text { Establishment of protein localization to } \\
\text { endoplasmic reticulum }\end{array}$ & $8 / 73$ & $\begin{array}{l}2.49 E \\
-08\end{array}$ & $\begin{array}{l}4.62 E \\
-06\end{array}$ & $\begin{array}{l}4.41 E \\
-06\end{array}$ & $\begin{array}{c}R P L 34, R P S 15 A, R P S 24, R P L 27, R P L 31, \\
\text { RPL35A, RPL17, RPS14 }\end{array}$ \\
\hline $\mathrm{BP}$ & 0019080 & Viral gene expression & $9 / 73$ & $\begin{array}{c}5.61 E \\
-08\end{array}$ & $\begin{array}{c}8.95 E \\
-06\end{array}$ & $\begin{array}{l}8.54 E \\
-06\end{array}$ & $\begin{array}{l}\text { RPL34, RPS15A, RPS24, RPL27, RPL31, } \\
\quad \text { RPL35A, RPL17, RPS14, DENR }\end{array}$ \\
\hline BP & 0019083 & Viral transcription & $8 / 73$ & $\begin{array}{c}4.43 E \\
-07\end{array}$ & $\begin{array}{l}4.49 E \\
-05\end{array}$ & $\begin{array}{l}4.29 E \\
-05\end{array}$ & $\begin{array}{c}\text { RPL34, } R P S 15 A, R P S 24, R P L 27, R P L 31, \\
\text { RPL35A, RPL17, RPS14 }\end{array}$ \\
\hline $\mathrm{CC}$ & 0022626 & Cytosolic ribosome & $9 / 74$ & $\begin{array}{c}3.61 E \\
-10\end{array}$ & $\begin{array}{l}6.28 E \\
-08\end{array}$ & $\begin{array}{l}5.81 E \\
-08\end{array}$ & $\begin{array}{l}\text { RPL34, RPS15A, RPS24, RPL27, RPL31, } \\
\quad \text { RPL35A, RSL24D1, RPL17, RPS14 }\end{array}$ \\
\hline $\mathrm{CC}$ & 0044445 & Cytosolic part & $9 / 74$ & $\begin{array}{l}3.57 E \\
-07\end{array}$ & $\begin{array}{l}1.55 E \\
-05\end{array}$ & $\begin{array}{l}1.44 E \\
-05\end{array}$ & $\begin{array}{l}\text { RPL34, RPS15A, RPS24, RPL27, RPL31, } \\
\quad \text { RPL35A, RSL24D1, RPL17, RPS14 }\end{array}$ \\
\hline MF & 0003735 & Structural constituent of ribosome & $9 / 73$ & $\begin{array}{c}1.42 E \\
-07\end{array}$ & $\begin{array}{l}2.54 E \\
-05\end{array}$ & $\begin{array}{c}2.45 E \\
-05\end{array}$ & $\begin{array}{l}\text { RPL34, RPS15A, RPS24, RPL27, RPL31, } \\
\quad \text { RPL35A, RSL24D1, RPL17, RPS14 }\end{array}$ \\
\hline
\end{tabular}

Abbreviation: BP: biological process; CC: cellular component; MF: molecular function.

supposed to be more important than others because of their key position on the PPI network. Key genes are shown in Table 4 and Figure 9. The expression of key genes in female IS patients based on follow-up vs. baseline is shown in Figure 10.

3.2. Discussion. Sex influence IS epidemiology, pathophysiology, treatment efficacy, and outcome. A recent clinical study found that minocycline, which is an inhibitor of cell death, had significantly better neurological outcomes in IS male patients only, with no significant clinical improvement to female patients [14]. The study illustrated the importance of assessing sex as a variable in IS clinical research. Recent years, microarray analyses have been attempted in sex differences of human IS. Some previous studies have screened sexually dimorphic differences of gene expression by comparing male IS patients with females directly [15]. However, some DEGs were located on sex chromosomes that could represent transcript expression differences based on sex or differences based on disease being studied. To eliminate the confounding factors of sex chromosomes, the study of Tian et al. adopted a method that female IS patients were compared with female controls, and male IS patients were compared with male controls. The focus of the study of Tian et al. was the sexually dimorphic differences of gene expression of peripheral blood total RNA in untreated stroke patients within 3 hours after acute IS onset without discussion about the sexually dimorphic differences of IS progression [16]. The sex differences of gene expression of peripheral blood total RNA underlying in IS progression remain poorly understood $[17,18]$.

Our study is aimed at identifying the sex-specific DEGs in peripheral blood in IS progression, predicting potential bio- markers that can lead to sex-specific peripheral immune response and prognosis, and revealing potential sex-specific treatment targets. The GSE37587 datasets included 68 peripheral whole blood samples which were collected 20 IS women and 14 IS men within 24 hours (baseline) from known onset of symptom and again at 24-48 hours (followup) after onset. In order to have further information whether the gene expression of sex-specific DEGs and nonsex-specific was consistent, we screened two kinds of DEGs: nonsex specific and sex specific. Through the follow-up of 34 patients compared with the baseline of 34 patients, we got the nonsex-specific DEGs of the progression of IS. Adopted method of Tian et al., which follow-up of 20 females were compared with baseline of 20 females and follow-up of 14 males compared with baseline of 14 males, respectively, we got the sex-specific DEGs of the progression post-IS. Interestingly, the results show that there are 30 nonsex-specific DEGs, 79 female-specific DEGs, and none of male-specific DEGs. There are 23 overlaps between female-specific and nonsex-specific DEGs (Figure 1). Only 7 genes are unique to nonsex-specific DEGs. The only one gene upregulated in nonsex DEGs is also the one of 25 upregulated in femalespecific DEGs (Table 1). It can be inferred that the femalespecific DEGs are contributed to the most of the nonsexspecific DEGs. Therefore, we only need to analyze the female-specific DEGs to know the sexually dimorphic in the early recovery of IS patients. More than two-thirds of the female-specific DEGs are downregulated during the course of 24-48 hours after stroke (Figures 2 and 3).

GO analysis showed that the 11 genes enriched were mainly related to ribosomal and protein synthesis. Changes in the transcription level of ribosome-related genes have a 


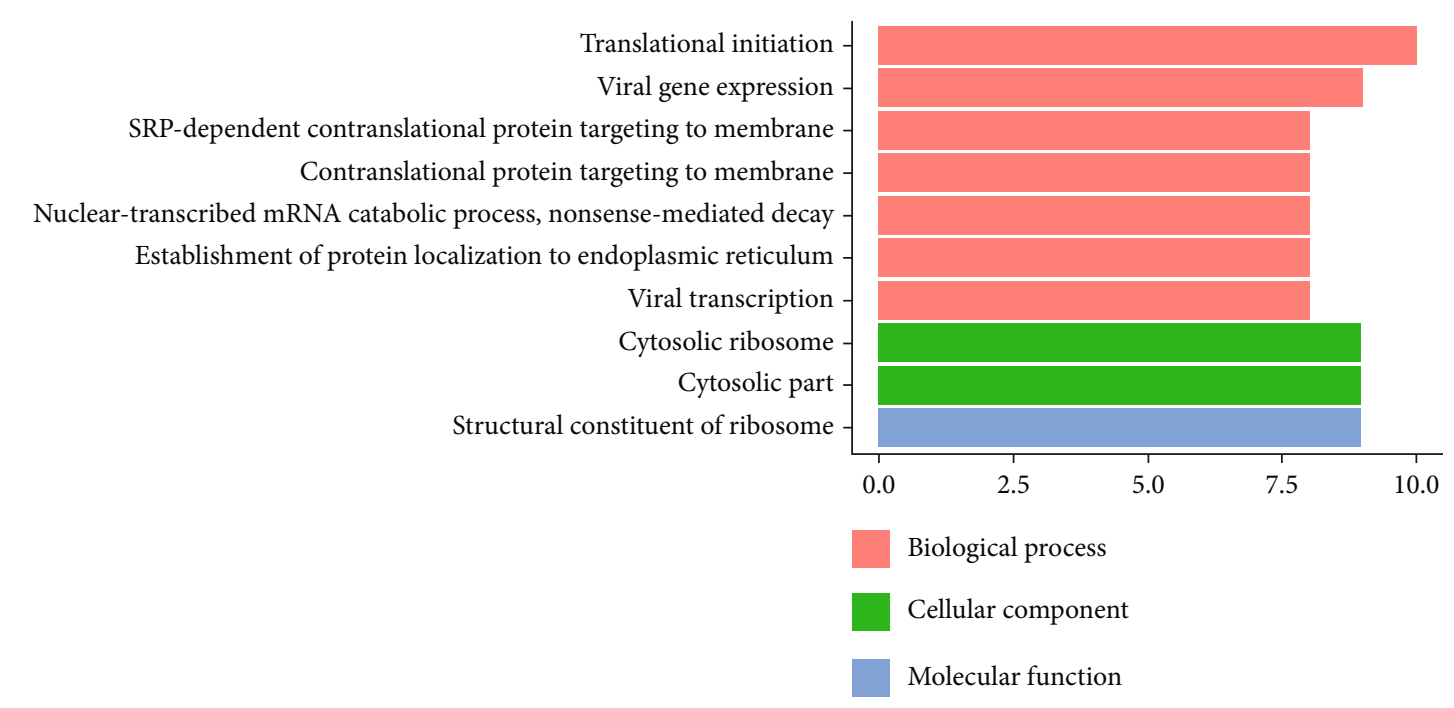

(a)

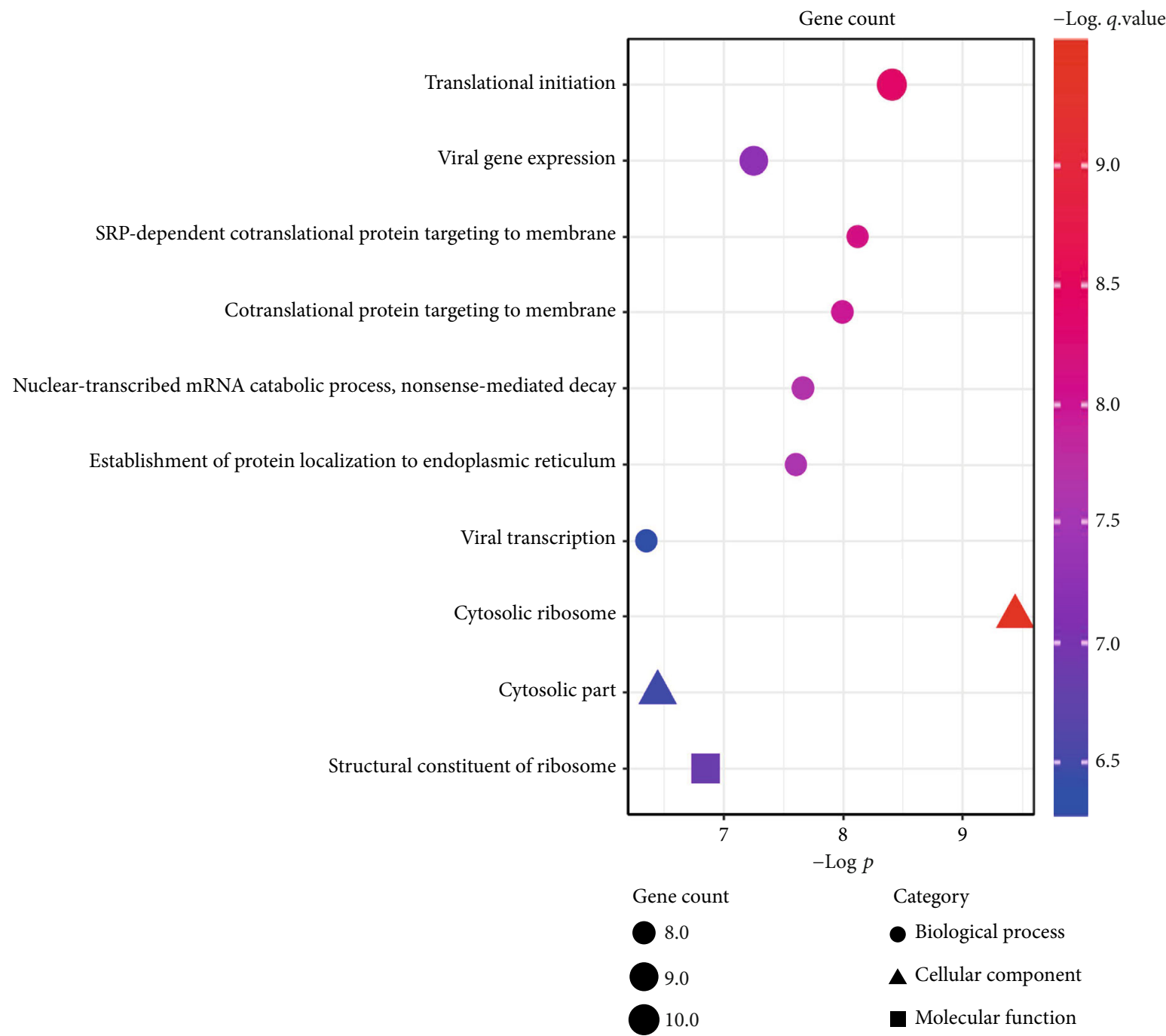

(b)

FIGURE 4: GO enrichment analysis of female-specific DEGs. (a) Gene count in biological process, cell component, and molecular function. (b) GO enrichment significance terms of female-specific DEGs in different functional groups. 


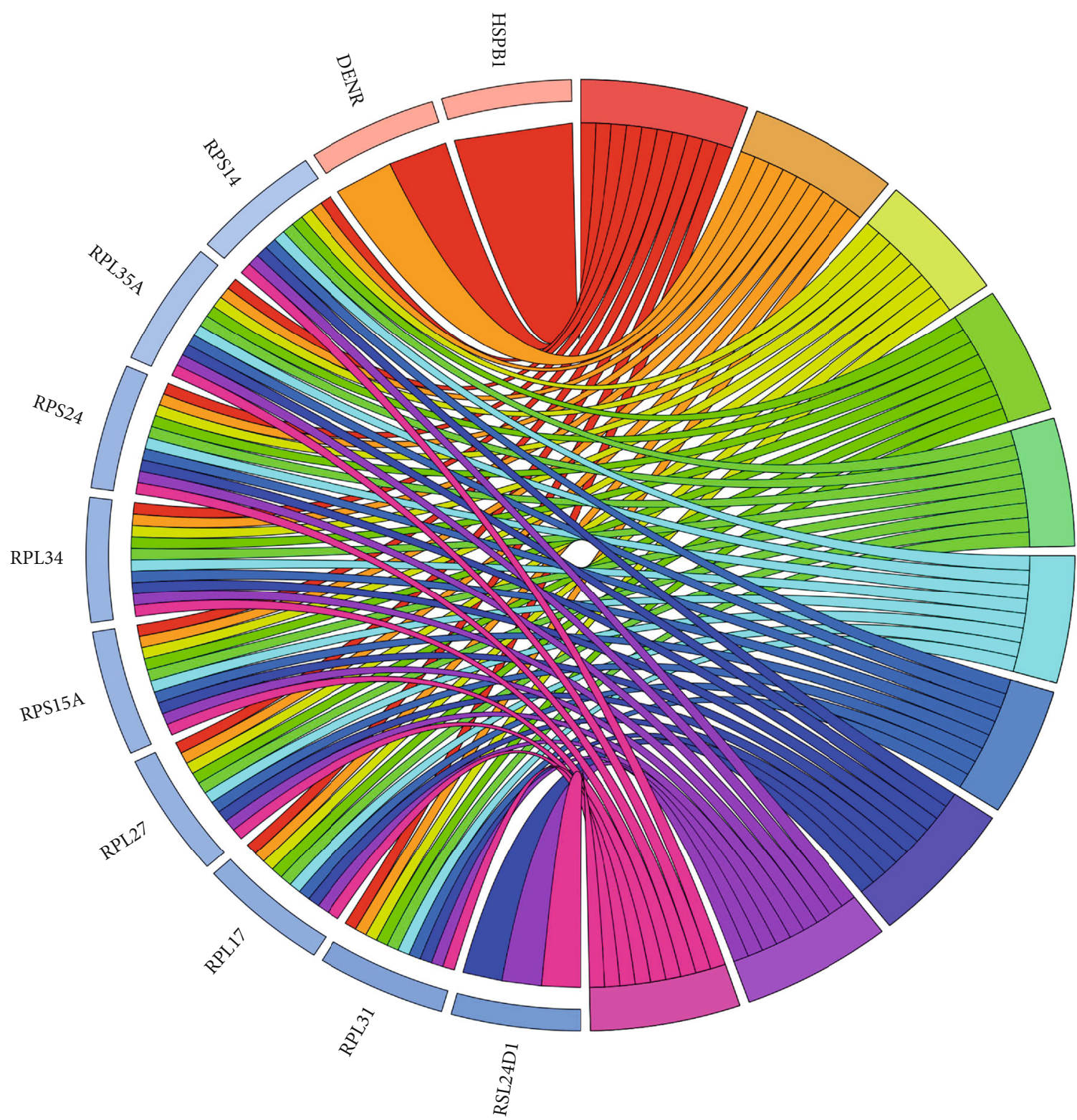

$\log _{2}$ FC

$-1$

\section{1}

$\square$ Translational initiation

$\square$ Nuclear-transcribed mRNA catabolic process, nonsense-mediated decay

Cytosolic part $\square$ Establishment of protein localization to endoplasmic reticulum

$\square$ Structural constituent of ribosome

$\square$ SRP-dependent cotranslational protein targeting to membrane
Viral transcription

$\square$ Cotranslational protein targeting to membrane

Cytosolic ribosome

$\square$ Viral gene expression

FIGURE 5: Distribution of female-specific DEGs in different GO enrichment functions.

broader impact on the downstream translation level. An in vitro experiment used ribosome profiling to explore the immediate transcription and translation change of neural cell gene expression to oxygen and glucose deprivation (OGD). It made a conclusion that the effect of OGD was widespread on translation than transcription [19]. It can be inferred that the 
TABLE 3: KEGG pathway enrichment analysis of female-specific DEGs.

\begin{tabular}{lcccccc}
\hline ID & Description & $\begin{array}{c}\text { Gene } \\
\text { ratio }\end{array}$ & BgRatio & $p$ value & $p$ adjust & Genes \\
\hline hsa03010 & Ribosome & $10 / 41$ & $153 / 8016$ & $3.23 E-09$ & $\begin{array}{c}3.48 E \\
-07\end{array}$ & RPL34, RPS15A, RPS24, RPL27, FAU, RPL31, RPL35A, \\
& NF-kappa B signaling & $3 / 41$ & $102 / 8016$ & 0.015027 & 0.54667 & RSL24D1, RPL17, RPS14 \\
hsa04064 & pathway & & & & LY96, BIRC2, IL8 \\
hsa04210 & Apoptosis & $3 / 41$ & $136 / 8016$ & 0.031862 & 0.54667 & DDIT3, BIRC2, CTSW \\
hsa04978 & Mineral absorption & $2 / 41$ & $58 / 8016$ & 0.035231 & 0.54667 & SLC40A1, CYBRD1 \\
hsa04932 & $\begin{array}{c}\text { Nonalcoholic fatty liver } \\
\text { disease (NAFLD) }\end{array}$ & $3 / 41$ & $149 / 8016$ & 0.040123 & 0.54667 & DDIT3, COX7B, IL8 \\
hsa05133 & Pertussis & $2 / 41$ & $76 / 8016$ & 0.057363 & 0.54667 & LY96, IL8 \\
\hline
\end{tabular}

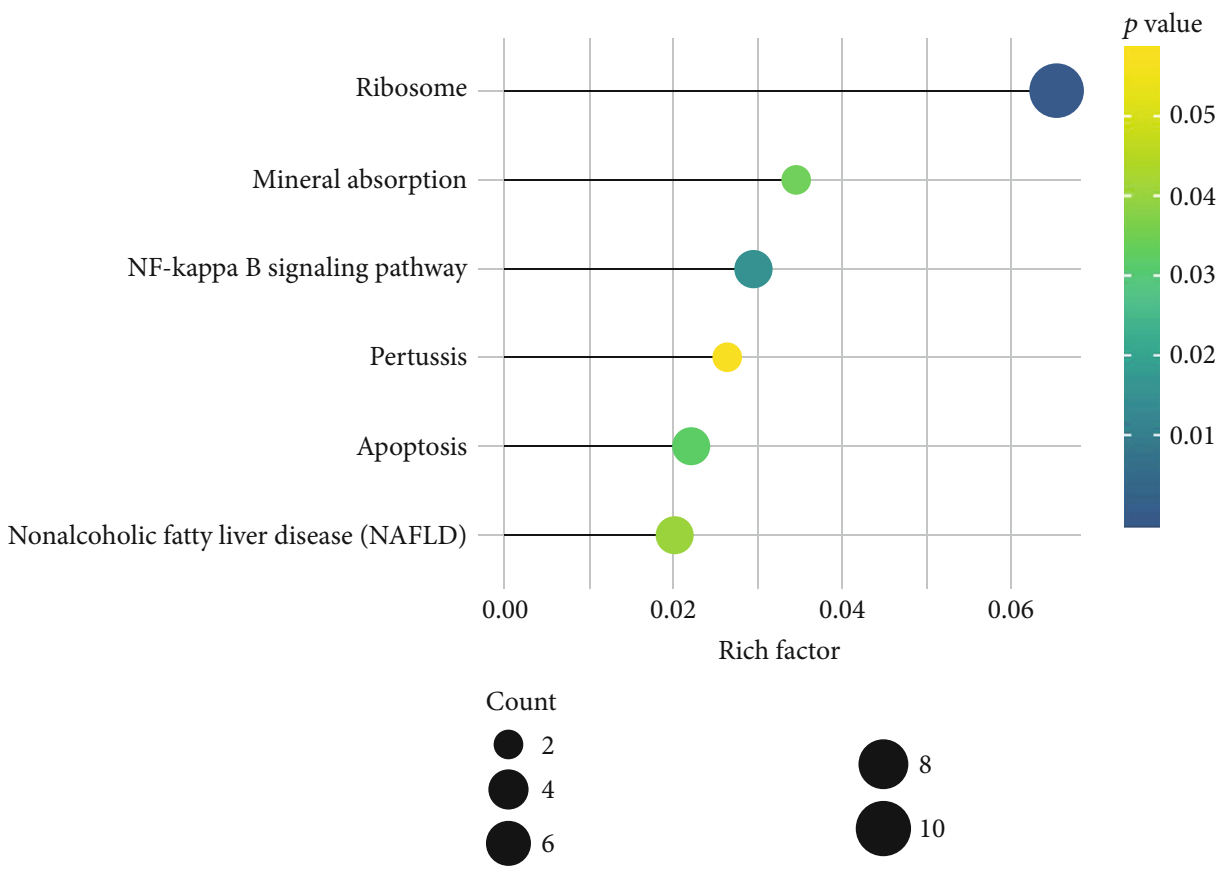

FIGURE 6: The top six enriched pathways with the highest rich factor of female-specific DEGs. Rich factor is equal to the gene count enriched into the pathway versus the total of genes contained in the pathway.

expression of sex-specific ribosome differential genes in the early stage of stroke recovery may be the key underlying mechanism of sexually dimorphic difference in immune response. In addition, although the change of ribosomerelated gene transcription level in peripheral blood reflects the peripheral immune response of granulocyte, lymphocytes, and monocytes to the greatest extent, there may also be genes coming from glial and nerve cells because of the damage of blood-brain barrier caused by stroke-induced inflammatory response. Therefore, the change of ribosomerelated gene expression may also reflect the repair mechanisms of damaged brain.

The results of the KEGG enrichment analysis of femalespecific DEGs are highly consistent with the GO analysis results. The most significant result is the ribosome followed by NF-kappa B signaling pathway and apoptosis and finally, the mineral absorption, nonalcoholic fatty liver disease, and pertussis. Ribosome is the place where biological protein synthesis takes place. It consists of two major components which the small ribosomal subunits in charge of reading the mRNA, and the large subunits in charge of joining amino acids to form a polypeptide chain. Each subunit consists of varieties of ribosomal proteins and one or more ribosomal RNA (rRNA) molecules. Ribosome-related genes downregulated lead to protein synthesis and secretion disorders. The NFkappa B signaling pathway has a broad role in the regulation of numerous biological processes. NF-kappa B-dependent gene expression is required for proper development and formation of the immune system, innate immune responses, initiation of the adaptive immune response, pathogen recognition, inflammation, lymphoid organogenesis, transcription of cytokines, and immediate antimicrobial responses [20-22]. Downregulation of NF-kappa B signaling pathway leads to immunosuppression $[23,24]$. Interestingly, 


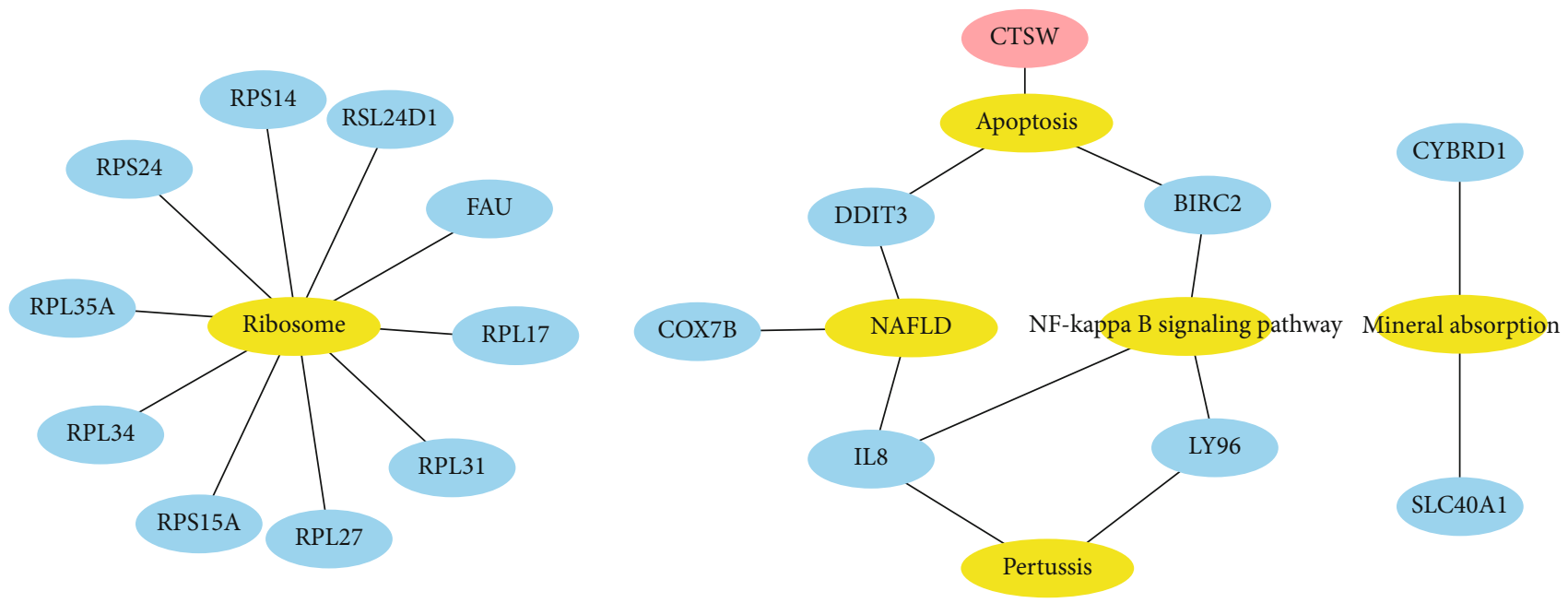

FIGURE 7: Significant pathway enrichment of female-specific DEGs. Yellow represents the signaling pathway, red represents the upregulated gene, and blue represents the downregulated gene. NAFLD: nonalcoholic fatty liver disease.

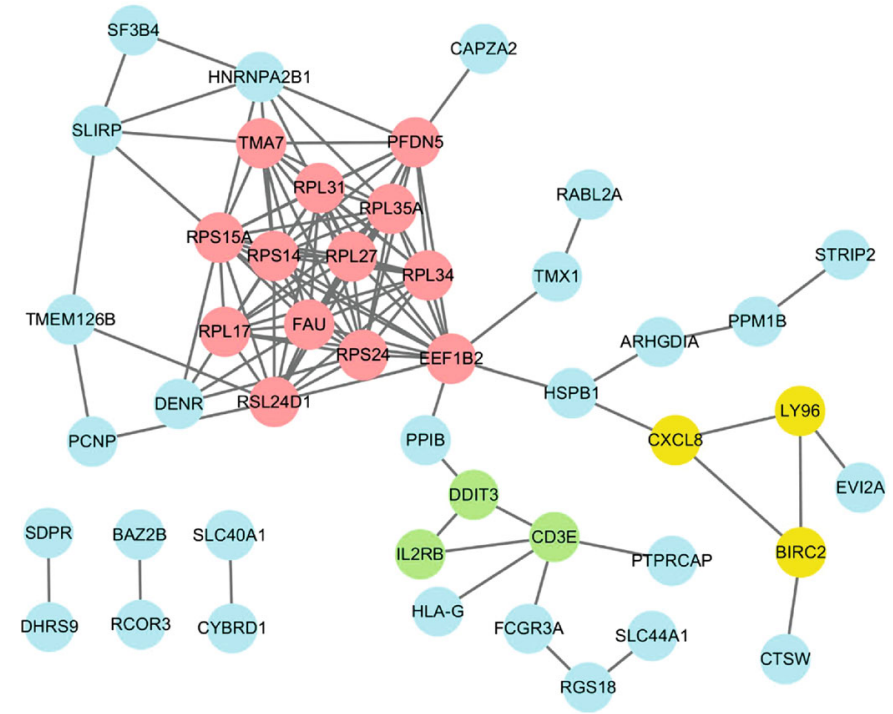

(a)

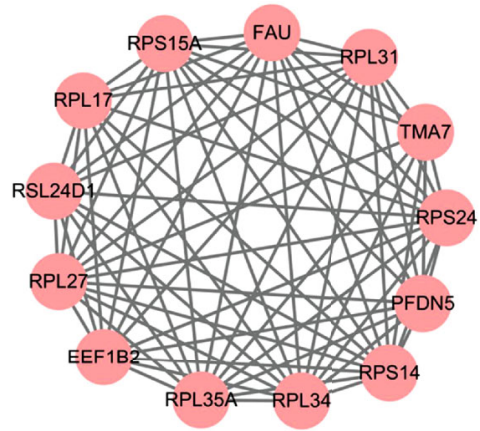

Cluster 1

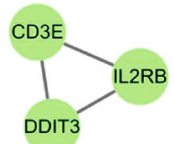

Cluster 2

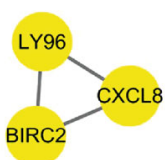

Cluster 3

(b)

Figure 8: (a) PPI network of female-specific DEGs. (b) Three functional modules. Red represents the cluster 1 with score $12.167,13$ nodes, and 73 edges. Green represents the cluster 2 with score 3, 3 nodes, and 3 edges. Yellow represents the cluster 3 with score 3, 3 nodes, and 3 edges. CXCL8 is also known as IL8.

we can see that the DDIT3 encoding a proapoptotic transcription factor DNA damage-inducible transcript 3 (DDIT3, also known as $\mathrm{CHOP}$ ) and the BIRC2 encoding cIAP1, which is a member of the inhibitor of apoptosis family that inhibits apoptosis [25], are both enriched in the apoptosis pathway with downregulated. One possible explanation of these is a tug of war between proapoptosis and inhibit apoptosis under the ribosome downregulated. In addition, the only one upregulated CTSW, encoding a cysteine proteinase associated with cytotoxic $\mathrm{T}$ and natural killer cell $[26,27]$, is enriched in the apoptosis pathway. We can infer that these results are likely associated with sexually dimorphic immune response, repair, or other complex mechanisms in IS progression.

Three modules were obtained from the functional module analysis of the PPI network. The clusters 1 and 3 are basically consistent with the results of KEGG enrichment analysis. The cluster 1 is closely related to ribosome function, and the important genes involved will be discussed in the following selection of key genes. The cluster 2 includes three genes, CD3E, IL2RB, and DDIT3. CD3e molecule, epsilon (also known CE3E), encoded by $C D 3 E$, 
TABle 4: Top 10 key genes in the PPI network of the female-specific DEGs ranked by MCC method.

\begin{tabular}{|c|c|c|c|c|}
\hline Rank & Gene symbol & Gene description & MCC score & $\log _{2} \mathrm{FC}$ \\
\hline 1 & RPS15A & Ribosomal protein $\mathrm{S} 15 \mathrm{a}$ & 4354708 & -0.83625 \\
\hline 2 & RPS14 & Ribosomal protein S14 & 4354704 & -0.63886 \\
\hline 3 & RPL27 & Ribosomal protein L27 & 4354680 & -0.84896 \\
\hline 3 & RPL35A & Ribosomal protein $\mathrm{L} 35 \mathrm{a}$ & 4354680 & -0.68986 \\
\hline 5 & FAU & FAU ubiquitin like and ribosomal protein $\mathrm{S} 30$ fusion & 4354584 & -0.72147 \\
\hline 5 & RPS24 & Ribosomal protein S24 & 4354584 & -0.81476 \\
\hline 7 & RPL31 & Ribosomal protein L31 & 4354560 & -1.0045 \\
\hline 7 & RPL34 & Ribosomal protein L34 & 4354560 & -0.82663 \\
\hline 9 & EEF1B2 & Eukaryotic translation elongation factor 1 beta 2 & 3991683 & -0.82928 \\
\hline 10 & RSL24D1 & Ribosomal L24 domain containing 1 & 3628802 & -1.0838 \\
\hline
\end{tabular}

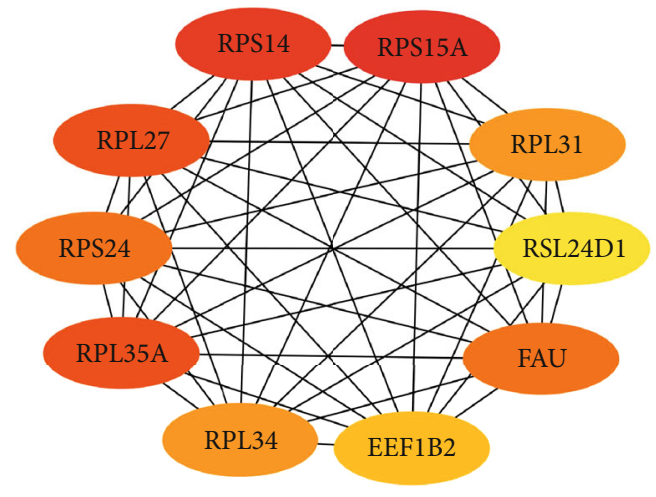

FIGURE 9: Top 10 key genes in the PPI network of the femalespecific DEGs.

together with CD3-gamma, -delta, and -zeta and the $\mathrm{T}$ cell receptor alpha/beta and gamma/delta heterodimers, forms the $\mathrm{T}$ cell receptor-CD3 complex, which couples antigen recognition to several intracellular signal-transduction pathways. CD3E plays an essential role in $\mathrm{T}$ cell development and interleukin-2 production in $T$ cells [28]. Interleukin-2 receptor subunit beta (also known as CD122), encoded by IL2RB, is involved in $\mathrm{T}$ cellmediated immune responses. Activation of the receptor increases proliferation of CD8+ effector T cells [29]. The downregulation of $C D 3 E$ and $I L 2 R B$ suggests the downregulation of $\mathrm{T}$ cell adaptive immune response. DDIT3 encodes DNA damage-inducible transcript 3 (DDIT3) that belongs to the family of CCAAT/enhancer-binding proteins (C/EBPs) [30]. It is also named C/EBP homologous protein $(\mathrm{CHOP})$, which can form heterodimers with other C/EBP family proteins [31]. DDIT3 plays an important role in endoplasmic reticulum (ER) stress-induced apoptosis [30]. Under ER stress, DDIT3 is a transcriptional repressor to downregulate the expression of antiapoptotic genes such as $B C L-2$ and a transcriptional activator to upregulate expression of proapoptotic genes such as $B A X$ $[30,32]$. Therefore, cluster 2 is related to $\mathrm{T}$ cell adaptive immune and apoptosis. The cluster 3 includes three genes, LY96, CXCL8 (also known as IL8), and BIRC2. The MD-2 protein (also known as lymphocyte antigen 96), encoded by $L Y 96$, associates with toll-like receptor 4 (TLR-4) on the cell surface and confers responsiveness to lipopolysaccharide (LPS) [33]. LPS is the ligand of TLR-4, and via p38, JNK, and NF-kappa B signaling pathways, its interaction results in activation of proinflammatory cytokines such as TNF $\alpha$, IL-1 $\beta$, IL-6, IL-18, and IL-12 [34] and chemokines such as IL8. IL8 (also known as CXCL8), encoded by CXCL8 (IL8), is a chemokine produced by macrophages mainly. Its primary function is to induce chemotaxis in target cells, primarily neutrophils, causing them to migrate toward the site of infection. cIAP1, encoded by BIRC2, is a member of the inhibitor of apoptosis family that inhibits apoptosis by interfering with the activation of caspases [35]. Meanwhile, cIAP1 also can set a balance between NF-kappa B transcription factor and apoptosis signaling downstream of tumor necrosis factor (TNF) receptor superfamily members by acting as ubiquitin E3 ligases for substrates that are part of the TNF receptor complex [36]. In addition, on the signalling route of TLR4- mediated production of proinflammatory cytokines, cIAPs are also required for ligand- induced degradation of TNF receptor-associated factor 3 (TRAF3), which functions as an inhibitor of mitogen-activated protein kinase (MAPK) activation and inflammatory cytokine production [37]. It can be concluded that the downregulation of the cluster 3 reveals the downregulation of NFkappa B signaling pathway, the reduction of cytokine, and decreased inhibition of apoptosis (Figure 11).

The results of key gene analysis further confirm the results of GO, KEGG, and functional module analysis. 10 top key genes screened, which all of them are downregulated, related to ribosome or translation. We obtained the basic information of these genes through the Human Gene Database GeneCards (https://www.genecards.org/). RPS14, RPS15A, and RPS24 encode 40S ribosomal proteins. FAU encodes a fusion protein consisting of the ubiquitin-like protein fubi at the $\mathrm{N}$ terminus and ribosomal protein S30 at the $\mathrm{C}$ terminus, which is posttranslationally processed to generate free fubi and free ribosomal protein S30. Whereas the function of fubi is currently unknown, ribosomal protein S30 is a component of the $40 \mathrm{~S}$ subunit of the cytoplasmic ribosome and displays antimicrobial activity [38]. RPL27, 

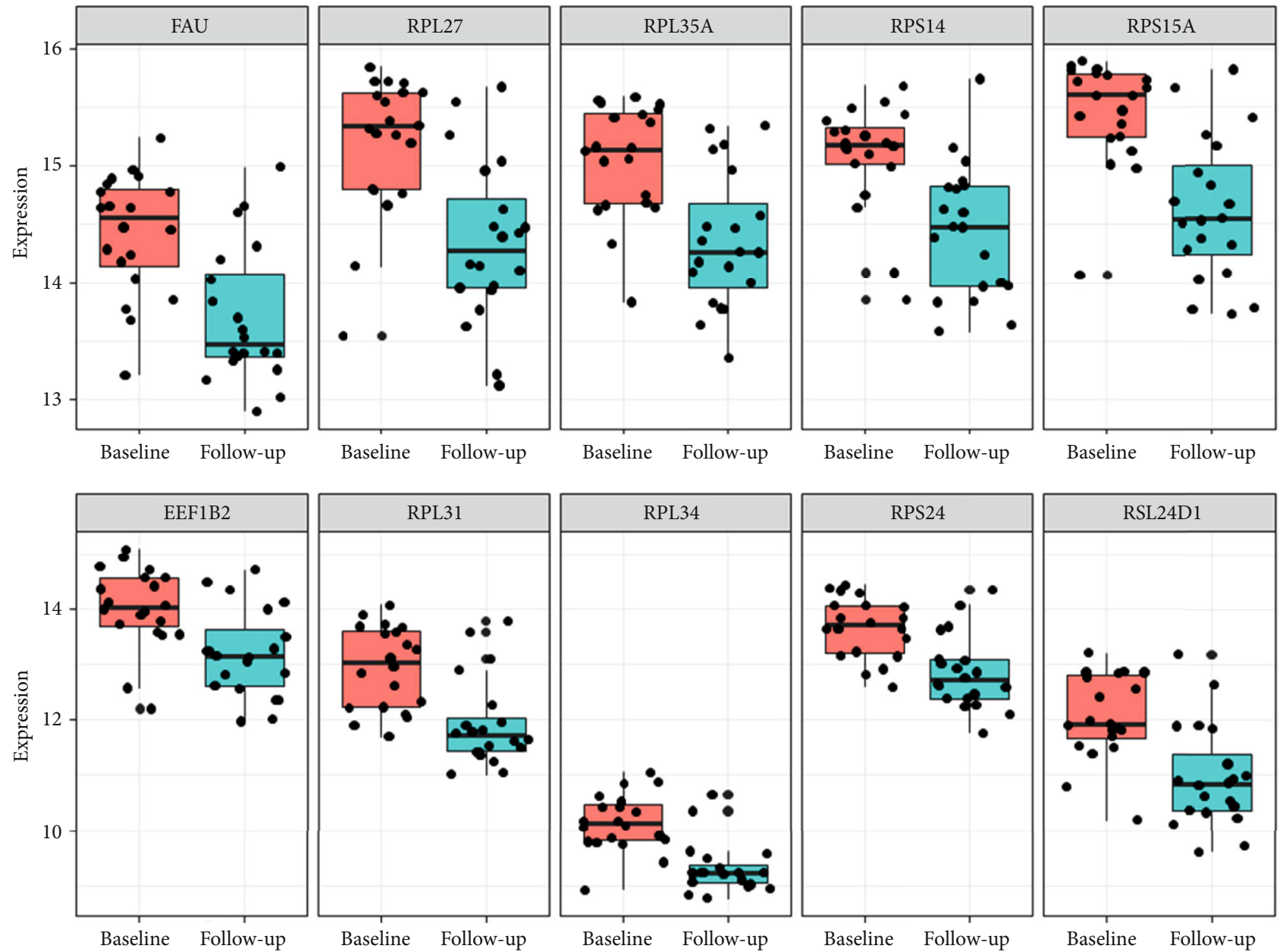

FIgURE 10: The expression of key genes in female IS patients based on follow-up vs. baseline.

RPL31, RPL34, and RPL35A encode 60S ribosomal proteins. The above key genes participate in the structure of ribosome and protein synthesis. RSL24D1 encodes probable ribosome biogenesis protein (RLP24), which localizes to the nucleolus and is thought to play a role in the biogenesis of the $60 \mathrm{~S}$ ribosomal subunit. EEF1B2 encodes a translation elongation factor, named eukaryotic translation elongation factor 1 beta 2 , which involved in the transfer of aminoacylated tRNAs to the ribosome. We infer that the downregulation of key genes will affect the protein synthesis of peripheral blood immune cells and thus cause immunosuppression.

As early as 1974, immunosuppression was found to be the most common complication of acute stroke [39]. Subsequently, a large number of studies showed that the main characteristics of SIDS are rapid and lasting immunosuppression: the decrease of monocyte activity, the decrease of T-lymphocyte count, the imbalance of Th1/Th2 [40], and the significant apoptosis of immune cells in peripheral immune organs (such as spleen and lymph nodes) [41, 42]. However, there are few reports about sexually dimorphic differences in SIDS. In this study, female IS patients show significant immunosuppressive response in the early recovery: ribosomal and NF-kappa B signaling pathway downregulation, $\mathrm{T}$ cell immune activity downregulation, and the level downregulation between proapoptosis and inhibit apoptosis, especially ribosome downregulation. However, the control male IS patients show few significant changes in peripheral gene expression. These may have an explanation why there are sex differences in stroke outcomes. At the same time, we observed the age of 20 female IS patients with mean age of $72.15 \pm 15.951$ years, which the youngest is 43 years and the oldest is 92 years. Most of them belong to the elderly women. Therefore, the occurrence of such differences may be more related to biological differences [43], rather than the differences caused by estrogen.

After a comprehensive exploring of female-specific DEGs, we studied main nonsex-specific DEGs. According to the introduction of GeneCards, RPL39 and RPL26 encode $60 \mathrm{~S}$ ribosomal proteins which work at the structure of ribosome and protein synthesis. The downregulation of these genes may affect the protein synthesis of peripheral blood immune cells. AKAP7 encodes a member of the A-kinase anchoring protein (AKAP) family, which are molecular scaffolding proteins binding to a regulatory subunit (RII) of cAMP-dependent protein kinase A (PKA), directing the kinase to discrete subcellular locations [44, 45]. Alternatively spliced transcript variants from the AKAP7 (AKAP15/18) are 


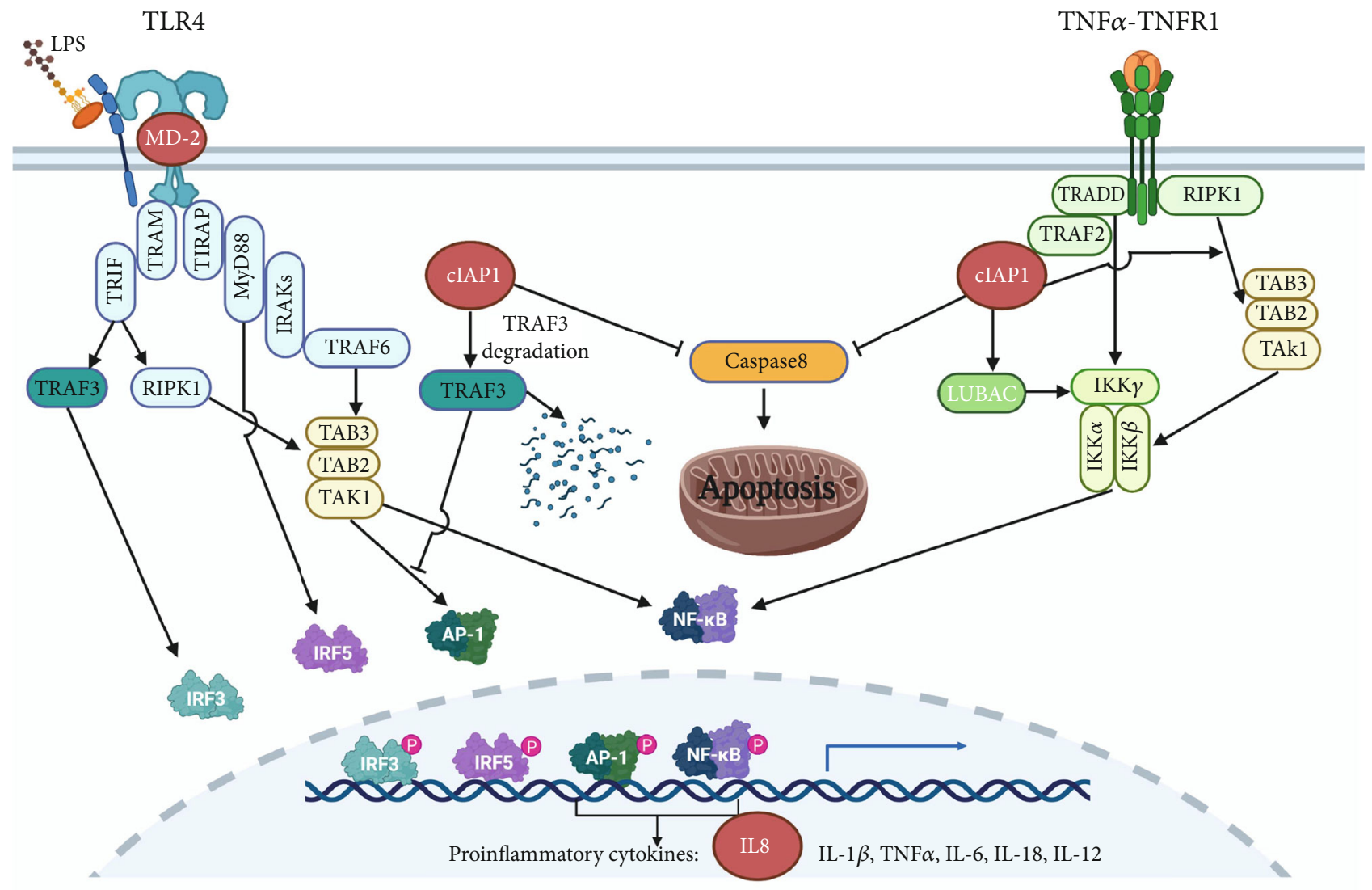

FIGURE 11: The mechanism of the cluster 3 DEGs in stroke-induced apoptosis and immunosuppression (created with http://BioRender.com/ ) .

essential components of neuronal and cardiac phosphatase complexes, ion channels, cardiac $\mathrm{Ca}^{2+}$ handling, and renal water transport [46]. A new study found that early heightened expression levels of $A K A P 7$ were significantly associated with poststroke blood-brain barrier (BBB) disruption 24 hours posthospital admission, which suggested that $A K A P 7$ may be a prognostic biomarker for poststroke BBB complications [47]. In this study, the downregulation of AKAP7 may indicate that the severity of BBB damage at post-IS 48 hours is lower than at 24 hours; in other word, it is within post-IS 48 hours that the BBB damage begins to repair. The role of other nonsex-specific DEGs on the development and prognosis of IS remains to be further studied.

The innovation of this study is to take the lead in analyzing the difference of gene expression between men and women in the early stage of stroke recovery through highthroughput biochip transcriptome datasets and to find that women are more responsive in stroke-induced immunosuppression, and ribosome-related genes play the most important roles in stroke-induced immunosuppression. This finding provides a new insight to evaluate the prognosis and potential accurate treatment targets. Ribosome-related genes may be a novel prognostic biomarker and a potential therapeutic target for IS. However, the specific mechanism of ribosome in SIDS needs to be further studied in vitro and in vivo.

\section{Conclusions}

Sex difference of ribosome in stroke-induced peripheral immunosuppression may be the potential mechanism of sex disparities in outcome after IS, and women are more likely to have SIDS after IS. RPS14, RPS15A, RPS24, FAU, RPL27, RPL31, RPL34, RPL35A, RSL24D1, and EEF1B2 may be novel prognostic biomarkers and potential therapeutic targets for IS.

\section{Data Availability}

The data used to support the findings of this study are included within the article.

\section{Conflicts of Interest}

The authors declare that there is no conflict of interest regarding the publication of this paper.

\section{Acknowledgments}

This study was supported by Gansu Province Science and Technology Plan Project (1506RJZA230) and Lanzhou University Second Hospital 2019 Special Fund for Doctoral Cultivation (YJS-BD-23). 


\section{References}

[1] C. O. Johnson, M. Nguyen, G. A. Roth et al., "Global, regional, and national burden of stroke, 1990-2016: a systematic analysis for the Global Burden of Disease Study 2016," The Lancet Neurology, vol. 18, no. 5, pp. 439-458, 2019.

[2] GBD 2016 Lifetime Risk of Stroke Collaborators, V. L. Feigin, G. Nguyen et al., "Global, regional, and country-specific lifetime risks of stroke, 1990 and 2016," The New England Journal of Medicine, vol. 379, no. 25, pp. 2429-2437, 2018.

[3] E. J. Benjamin, P. Muntner, A. Alonso et al., "Heart Disease and Stroke Statistics-2019 update: a report from the American Heart Association," Circulation, vol. 139, no. 10, pp. e56-e528, 2019.

[4] D. W. Brann, K. Dhandapani, C. Wakade, V. B. Mahesh, and M. M. Khan, "Neurotrophic and neuroprotective actions of estrogen: basic mechanisms and clinical implications," Steroids, vol. 72, no. 5, pp. 381-405, 2007.

[5] A. Banerjee, J. Wang, S. Bodhankar, A. A. Vandenbark, S. J. Murphy, and H. Offner, "Phenotypic changes in immune cell subsets reflect increased infarct volume in male vs. female mice," Translational Stroke Research, vol. 4, no. 5, pp. 554563, 2013.

[6] T. Gattringer, J. Ferrari, M. Knoflach et al., "Sex-related differences of acute stroke unit care: results from the Austrian stroke unit registry," Stroke, vol. 45, no. 6, pp. 1632-1638, 2014.

[7] F. H. Spaander, S. M. Zinkstok, I. M. Baharoglu et al., "Sex differences and functional outcome after intravenous thrombolysis," Stroke, vol. 48, no. 3, pp. 699-703, 2017.

[8] E. Choleris, L. A. M. Galea, F. Sohrabji, and K. M. Frick, "Sex differences in the brain: implications for behavioral and biomedical research," Neuroscience and Biobehavioral Reviews, vol. 85, pp. 126-145, 2018.

[9] K. Prass, C. Meisel, C. Höflich et al., "Stroke-induced immunodeficiency promotes spontaneous bacterial infections and is mediated by sympathetic activation reversal by poststroke $\mathrm{T}$ helper cell type 1-like immunostimulation," The Journal of Experimental Medicine, vol. 198, no. 5, pp. 725-736, 2003.

[10] M. Preininger, D. Arafat, J. Kim et al., "Blood-informative transcripts define nine common axes of peripheral blood gene expression," PLoS Genetics, vol. 9, no. 3, article e1003362, 2013.

[11] K. Musunuru, E. Ingelsson, M. Fornage et al., "The expressed genome in cardiovascular diseases and stroke: refinement, diagnosis, and prediction: a scientific statement from the American Heart Association," Circulation: Cardiovascular Genetics, vol. 10, no. 4, article e4000037, 2017.

[12] S. Falcione, J. Kamtchum-Tatuene, G. Sykes, and G. C. Jickling, "RNA expression studies in stroke: what can they tell us about stroke mechanism?," Current Opinion in Neurology, vol. 33, no. 1, pp. 24-29, 2020.

[13] G. Yu, L. G. Wang, Y. Han, and Q. Y. He, "clusterProfiler: an R package for comparing biological themes among gene clusters," OMICS, vol. 16, no. 5, pp. 284-287, 2012.

[14] M. R. Amiri-Nikpour, S. Nazarbaghi, M. Hamdi-Holasou, and Y. Rezaei, "An open-label evaluator-blinded clinical study of minocycline neuroprotection in ischemic stroke: genderdependent effect," Acta Neurologica Scandinavica, vol. 131, no. 1, pp. 45-50, 2015.

[15] W. Zhu, Y. Nan, S. Wang, and W. Liu, "Bioinformatics analysis of gene expression profiles of sex differences in ischemic stroke," BioMed Research international, vol. 2019, Article ID 2478453, 7 pages, 2019.

[16] Y. Tian, B. Stamova, G. C. Jickling et al., "Effects of gender on gene expression in the blood of ischemic stroke patients," Journal of Cerebral Blood Flow and Metabolism: Official Journal of the International Society of Cerebral Blood Flow and Metabolism, vol. 32, no. 5, pp. 780-791, 2012.

[17] W. X. Li, S. X. Dai, Q. Wang et al., "Integrated analysis of ischemic stroke datasets revealed sex and age difference in anti-stroke targets," PeerJ, vol. 4, article e2470, 2016.

[18] B. Stamova, G. C. Jickling, B. P. Ander et al., "Gene expression in peripheral immune cells following cardioembolic stroke is sexually dimorphic," PloS One, vol. 9, no. 7, article e102550, 2014.

[19] D. E. Andreev, P. B. F. O'Connor, A. V. Zhdanov et al., "Oxygen and glucose deprivation induces widespread alterations in mRNA translation within 20 minutes," Genome Biology, vol. 16, no. 1, 2015.

[20] Q. Zhang, M. J. Lenardo, and D. Baltimore, "30 years of NF$\kappa \mathrm{B}$ : a blossoming of relevance to human pathobiology," Cell, vol. 168, no. 1-2, pp. 37-57, 2017.

[21] M. C. Mulero, T. Huxford, and G. Ghosh, "NF- $\kappa$ B, I $\kappa$ B, and IKK: integral components of immune system signaling," Advances in Experimental Medicine and Biology, vol. 1172, pp. 207-226, 2019.

[22] M. S. Hayden, A. P. West, and S. Ghosh, "NF- $\kappa$ B and the immune response," Oncogene, vol. 25, no. 51, pp. 6758-6780, 2006.

[23] Q. W. Deng, H. Yang, F. L. Yan et al., "Blocking sympathetic nervous system reverses partially stroke-induced immunosuppression but does not aggravate functional outcome after experimental stroke in rats," Neurochemical Research, vol. 41, no. 8, pp. 1877-1886, 2016.

[24] L. Zuo, L. Shi, and F. Yan, "The reciprocal interaction of sympathetic nervous system and cAMP-PKA-NF-kB pathway in immune suppression after experimental stroke," Neuroscience Letters, vol. 627, pp. 205-210, 2016.

[25] P. Listen, N. Roy, K. Tamai et al., "Suppression of apoptosis in mammalian cells by NAIP and a related family of IAP genes," Nature, vol. 379, no. 6563, pp. 349-353, 1996.

[26] T. Wex, F. Bühling, H. Wex et al., "Human cathepsin W, a cysteine protease predominantly expressed in NK cells, is mainly localized in the endoplasmic reticulum," Journal of Immunology, vol. 167, no. 4, pp. 2172-2178, 2001.

[27] R. I. Brinkworth, J. F. Tort, P. J. Brindley, and J. P. Dalton, "Phylogenetic relationships and theoretical model of human cathepsin W (lymphopain), a cysteine proteinase from cytotoxic T lymphocytes," The International Journal of Biochemistry \& Cell Biology, vol. 32, no. 3, pp. 373-384, 2000.

[28] T. Yamazaki, Y. Hamano, H. Tashiro et al., "CAST, a novel $\mathrm{CD} 3 \varepsilon$-binding protein transducing activation signal for interleukin-2 production in T cells," The Journal of Biological Chemistry, vol. 274, no. 26, pp. 18173-18180, 1999.

[29] O. Boyman and J. Sprent, "The role of interleukin-2 during homeostasis and activation of the immune system," Nature Reviews. Immunology, vol. 12, no. 3, pp. 180-190, 2012.

[30] H. Hu, M. Tian, C. Ding, and S. Yu, “The C/EBP homologous protein (CHOP) transcription factor functions in endoplasmic reticulum stress-induced apoptosis and microbial infection," Frontiers in Immunology, vol. 9, 2019.

[31] M. Ubeda, X. Z. Wang, H. Zinszner, I. Wu, J. F. Habener, and D. Ron, "Stress-induced binding of the transcriptional factor 
CHOP to a novel DNA control element," Molecular and Cellular Biology, vol. 16, no. 4, pp. 1479-1489, 1996.

[32] H. Tsukano, T. Gotoh, M. Endo et al., "The endoplasmic reticulum stress-C/EBP homologous protein pathway-mediated apoptosis in macrophages contributes to the instability of atherosclerotic plaques," Arteriosclerosis, Thrombosis, and Vascular Biology, vol. 30, no. 10, pp. 1925-1932, 2010.

[33] F. Re and J. L. Strominger, "Monomeric recombinant MD-2 binds toll-like receptor 4 tightly and confers lipopolysaccharide responsiveness," The Journal of Biological Chemistry, vol. 277, no. 26, pp. 23427-23432, 2002.

[34] S. Siegemund and K. Sauer, "Balancing pro- and antiinflammatory TLR4 signaling," Nature Immunology, vol. 13, no. 11, pp. 1031-1033, 2012.

[35] M. Rothe, M. G. Pan, W. J. Henzel, T. M. Ayres, and D. V. Goeddel, "The TNFR2-TRAF signaling complex contains two novel proteins related to baculoviral inhibitor of apoptosis proteins," Cell, vol. 83, no. 7, pp. 1243-1252, 1995.

[36] P. D. Mace, C. Smits, D. L. Vaux, J. Silke, and C. L. Day, "Asymmetric recruitment of cIAPs by TRAF2," Journal of Molecular Biology, vol. 400, no. 1, pp. 8-15, 2010.

[37] M. Gyrd-Hansen and P. Meier, "IAPs: from caspase inhibitors to modulators of NF-kappaB, inflammation and cancer," Nature Reviews. Cancer, vol. 10, no. 8, pp. 561-574, 2010.

[38] K. Kas, L. Michiels, and J. Merregaert, "Genomic structure and expression of the human fau gene: encoding the ribosomal protein S30 fused to a ubiquitin-like protein," Biochemical and Biophysical Research Communications, vol. 187, no. 2, pp. 927-933, 1992.

[39] R. J. Howard and R. L. Simmons, "Acquired immunologic deficiencies after trauma and surgical procedures," Surgery, Gynecology \& Obstetrics, vol. 139, no. 5, pp. 771-782, 1974.

[40] K. G. Haeusler, W. U. Schmidt, F. Föhring et al., "Cellular immunodepression preceding infectious complications after acute ischemic stroke in humans," Cerebrovascular Diseases, vol. 25, no. 1-2, pp. 50-58, 2008.

[41] A. Liesz, S. Hagmann, C. Zschoche et al., "The spectrum of systemic immune alterations after murine focal ischemia: immunodepression versus immunomodulation," Stroke, vol. 40, no. 8, pp. 2849-2858, 2009.

[42] F. L. Yan and J. H. Zhang, "Role of the sympathetic nervous system and spleen in experimental stroke-induced immunodepression," Medical Science Monitor, vol. 20, pp. 2489-2496, 2014.

[43] B. Stamova, Y. Tian, G. Jickling et al., "The X-chromosome has a different pattern of gene expression in women compared with men with ischemic stroke," Stroke, vol. 43, no. 2, pp. 326-334, 2012.

[44] I. D. Fraser, S. J. Tavalin, L. B. Lester et al., "A novel lipidanchored A-kinase anchoring protein facilitates cAMPresponsive membrane events," The EMBO Journal, vol. 17, no. 8, pp. 2261-2272, 1998.

[45] E. C. Greenwald, J. M. Redden, K. L. Dodge-Kafka, and J. J. Saucerman, "Scaffold state switching amplifies, accelerates, and insulates protein kinase C signaling," The Journal of Biological Chemistry, vol. 289, no. 4, pp. 2353-2360, 2014.
[46] K. R. Johnson, J. Nicodemus-Johnson, G. K. Carnegie, and R. S. Danziger, "Molecular evolution of A-kinase anchoring protein (AKAP)-7: implications in comparative PKA compartmentalization," BMC Evolutionary Biology, vol. 12, no. 1, p. 125, 2012.

[47] G. C. O'Connell, M. B. Treadway, A. B. Petrone et al., "Peripheral blood AKAP7 expression as an early marker for lymphocyte-mediated post-stroke blood brain barrier disruption," Scientific Reports, vol. 7, no. 1, p. 1172, 2017. 